\title{
Hematological Malignancies causing intestinal obstruction-Rare cases
}

\author{
Prof.S.Subbiah MS.Mch ${ }^{1}$, Prof.G.Gopu MS.Mch ${ }^{1}$, Dr.Sujay Susikar MS.Mch², \\ Dr.R.S.Bright Singh,resident ${ }^{2}$. \\ ${ }^{1,2}$ Department of Surgical Oncology, Govt. Royapettah Hospital, Kilpauk Medical College, Chennai, India.
}

\begin{abstract}
Hematological malignancies arise from bone marrow derived cells. They can be broadly classified as those of myeloid cell lines, neoplasm of lymphoid cell lines, lymphoproliferative disorders and Histolytic/Dendritic cell neoplasm. Hematological malignancies presenting with intestinal obstruction are rare. Only a few cases have been reported worldwide. We report two rare cases of hematological malignancies presenting with intestinal obstruction. A 55-year-old female, a case of secondary extra medullary plasmacytoma presented with obstruction due to small bowel intussusception and a 56-year-old male, presented with intestinal obstruction due to primary Non Hodgkin's lymphoma of the small bowel.
\end{abstract}

Keywords: Hematological malignancies, intestinal obstruction.

\section{Introduction}

Hematological malignancies presenting with intestinal obstruction are rare. Few cases are reported in the literature. Hematological malignancies involving small bowel are rare. Here we report two rare cases of hematological disorders presenting with intestinal obstruction.

Case Report 1: A case of Secondary Extra Medullary plasmacytoma presented with small bowel intussusception. A 55 year-old woman was evaluated for swelling in right parietal region 2 years ago and diagnosed with multiple myeloma. She was treated with bortezomib, dexamethasone and stem cell transplantation. She was disease free for 15 months. She developed sub acute intestinal obstruction after that for which laparotomy was done and ileo-ileal intussusception was found[Fig:1]. The affected segment was resected with mesentery and a primary end to end anastomoses was performed.On cut sections, it showed grossly circumferential tumor with fish flesh like cut surface[Fig:2]. Microscopic features showed typical and atypical plasma cells with a diagnosis of plasmacytoma (extramedullary arising in ileum)[Fig:3].

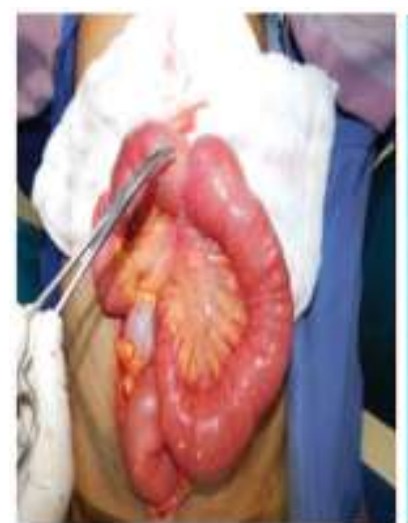

Fig:1 Ileo-ileal intussusception

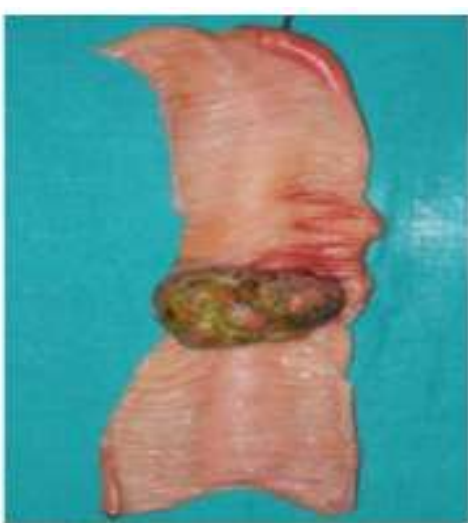

Fig:2 Cut open specimen

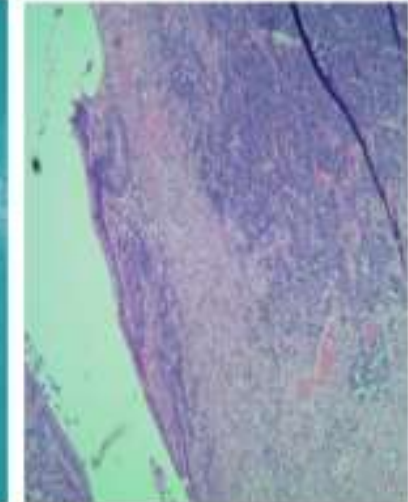

Fig:3 Microscopic image plasmacytoma

Case Report 2: A case of Primary Non Hodgkin's lymphoma presents with intestinal obstruction. A 56-year-old male presented with pain abdomen and vomiting of four days duration with sub acute intestinal obstruction. On abdominal examination a firm mobile mass of size $6 \times 8 \mathrm{~cm}$ was seen in the umbilical and right iliac fossa, no evidence of ascites and hepatosplenomegaly. The laboratory tests were normal except for anemia. A CT scan of abdomen and pelvis showed mass arising from the bowel. On laparotomy, there was a huge mesenteric mass of size $10 \times 8 \mathrm{~cm}$ involving the terminal ileum and caecum. Right hemicolectomy was done[Fig:4]. Gross cut section showed extramural mass with a small ulcero proliferative lesion in the lumen of the ileum. Histopathology revealed small bowel Non Hodgkin's lymphoma-diffuse large 
cell variant[Fig:5].Fourteen regional lymph nodes showed lymphomatous infiltrate. Bone marrow biopsy was done which was normal.

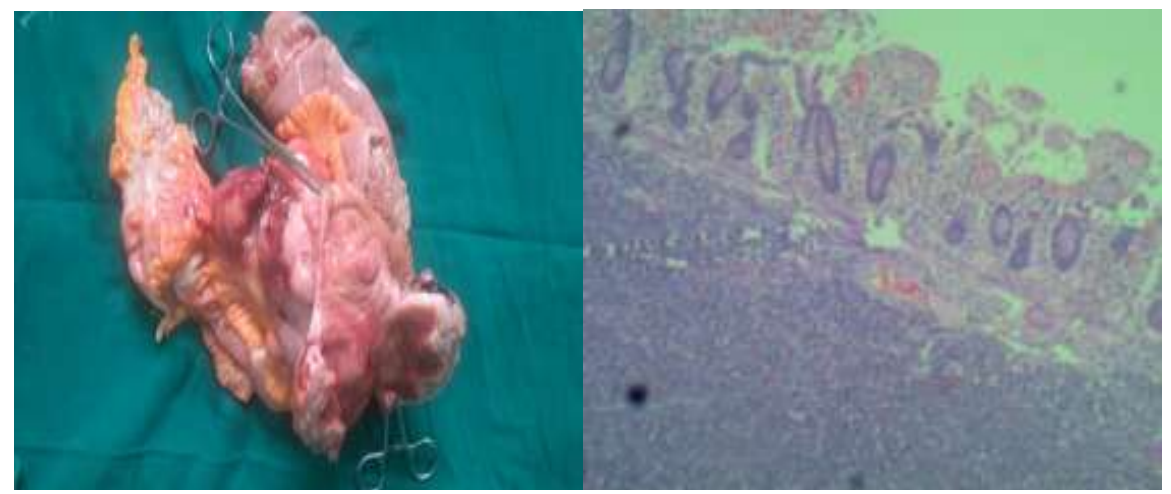

Fig:4 Specimen of right hemicolectomy Fig:5 Microscopic image of Non Hodgkin's lymphoma

\section{Discussion}

Plasma cell neoplasm is a type of hematological malignancy arising as a result of a monoclonal proliferation of plasma cells (lymphoid cell line) associated with the production of monoclonal proteins (M proteins). Malignant plasmablastic clones reside in the bone marrow. When on a rare occasion they migrate into nonmarrow soft tissue, it is termed as an extramedually plasmacytoma. This constitutes 3 - $4 \%$ of plasma cell neoplasms. Extramedullary plasmacytoma can present as a primary tumor or secondary to disseminated plasma cell neoplasm such as multiple myeloma. ${ }^{[1]}$ In secondary plasmacytoma (Multiple Myeloma), the involvement of gastrointestinal tract was found in fewer than $5 \%$ of autopsies of patients who died of complications of multiple myeloma. ${ }^{[2]}$ In large retrospective study, in 2584 multiple myeloma patients only $24(0.9 \%)$ of patients with gastrointestinal tract involvement were identified as Talamo et al ${ }^{[3]}$ Only 17 cases of extramedullary plasmacytoma affecting ileum have been reported in English literature, almost all primary in nature, only 3 primary cases of extramedullary plasmacytoma secondary to multiple myeloma reported. ${ }^{[4,5]}$ Intestinal obstruction caused by myeloma is rare. Only a few cases are on record as being diagnosed radiologically before patient's death. ${ }^{[3]}$ The prognosis of secondary extramedullary plasmacytoma is unknown. Even the small number of cases reported in literature showed poor prognosis. ${ }^{[6]}$ Radiotherapy or surgery alone is insufficient and systemic therapy is required. ${ }^{[1]}$ Systemic chemotherapy and autologous hemopoietic cell transplantation play a role. ${ }^{[3]}$ The role of surgery is often palliative to deal with resolvable life-threatening emergencies and possibly to prolong life.

Gastrointestinal lymphomas constitute $15-20 \%$ of all malignancies. Lymphoma can arise from any part of gastrointestinal tract. Gastrointestinal lymphomas are mostly of Non Hodgkin's type. The common sites involved are stomach (50-60\%) and small bowel (20-30\%). Among the small bowel, 60-65\% originate in the ileum, $20-25 \%$ from jejunum and 6-8\% in the duodenum. ${ }^{[7]}$ The terminal ileum is the most common site for lymphomas due to a higher concentration of lymphoid tissue ${ }^{[8]}$ Histologically small bowel lymphomas are divided into B-cell lymphomas [90\%] and T-cell lymphomas. T-cell lymphomas are rare, multifocal and most commonly affect proximal jejunum and are associated with celiac disease. Small bowel lymphomas may be primary or secondary. Primary small bowel lymphomas are very rare with only a few cases being reported. Primary small bowel lymphomas first involve the small intestine and later involve other parts of the body including lymph nodes and bone marrow. Secondary small bowel lymphomas arise from peripheral lymph nodes, bone marrow, and other organs, small intestinal involvement occurring latter. ${ }^{[9]}$ The distinction between primary and secondary infiltration of the gastrointestinal tract can be made by biopsy of bone marrow and imaging including a PET-CT. When this is not possible Dawson's criteria can be used. The criteria include 1) absence of peripheral lymphadenopathy at the time of presentation, 2) lack of enlarged mediastinal lymph nodes, 3) normal total and differential whole blood count, 4) predominance of bowel lesion at the time of laparotomy with only regional lymph node ,5) no lymphomatous involvement of liver and spleen ${ }^{[10]}$ The various (histological) subtypes of small intestinal lymphomas are mucosa associated lymphoid tissue (MALT), diffuse large B-cell lymphoma, follicular lymphoma, Burkitt's lymphoma and mantle cell lymphoma. Diffuse large Bcell Lymphoma constitutes $31 \%$ of Non Hodgkin's lymphoma there is slight male predominance. The median age is 64 years and the tumor express B-cell antigens. ${ }^{[1]}$ Due to the paucity of cases reported and absence of randomized trials in primary small bowel lymphoma, appropriate management of primary small bowel lymphoma is still unclear. The extent of surgery and whether the surgery delays chemotherapy is unclear. If the primary small bowel is diagnosed before or at laparotomy, surgical resection should be preferred in order to reduce the risk of serious complications such as perforation, bleeding, and obstruction. Low grade Non 
Hodgkin's lymphoma has a relatively good prognosis with a median survival as long as 10 years, however they are usually not curable when advanced. Intermediate and high-grade lymphomas are more aggressive but are more responsive to chemotherapy. ${ }^{[11}$ In some reports, surgery in combination with chemotherapy had a significant effect on survival in localized and in disseminated disease. The benefit of adding radiation remains unclear. ${ }^{[12,13,14,15]}$

\section{Conclusion}

Hematological malignancies presenting as intestinal obstruction are rare. Signs and symptoms are vague, which makes early diagnosis and treatment more difficult. However, these conditions should be remembered and considered in differential diagnosis of intestinal obstruction.

\section{References}

[1]. Arun A, Nick G, Jacob A. Secondary extramedullaryplasmacytoma causing small bowel intussusception in a patient with multiple myeloma- A case report. Int J Surg Case Rep.2013; 4(5): 486-488.

[2]. Telakis E, Tsironi E, Tavoularis G, Papatheodorou K, TzaidaO, Nikolaou A. Gastrointestinal involvement in a patient with multiple myeloma: A case report. Annals of gastroenterology. 2009;22(4):287-290.

[3]. Hill S.A, Yudelman P.L. Multiple myelomatosis: an unusual cause of small bowel intussusception. Postgraduate medical journal 1986; 62, 1043-1044.

[4]. Amanda E, Darren K, Jameson F, Ivan D. Plasmacytoma-Associated Obstruction of the small intestine in a Patient with multiple myeloma. Hospital Physician.Sept 2002.

[5]. Diana T, Stuart M, Catherine C, Ioannis G, Jagdish A, Ayub A.B, Neil S, Margaret R. Nonsecretory multiple myeloma presenting as an intestinal tumor. Case reports in hematology. volume 2015.

[6]. Dzierzanowski M, Porter G.A. An unusual presentation of a secondary extramedullaryplasmacytoma in a patient with multiple myeloma. Canadian Journal of Surgery. 2005;48:75-76.

[7]. Yin L, Chen C, Peng C, Chen G, Zhou H. Primary small-bowel non-Hodgkin's lymphoma: a study of clinical features, Pathology, Management and prognosis. The Journal of International Medical Research.2007;35:406-415

[8]. Diffuse large B-cell lymphoma (DLBCL): ESMO Clinical Practice Guidelines for diagnosis, treatment and follow-up; European Society for Medical Oncology (2015)

[9]. www.dovemed.com/diseases-conditions/lymphoma-small-intestine

[10]. Marcelo P , Adriana B, Leticia V , Francisco D , Joao G . Non-Hodgkin lymphoma as a cause of acute intestinal obstruction/perforation in patients with adenocarcinoma of the sigmoid colon: a case report. Journal of Coloproctology. October/December, 2011. Volume 31, No.4.

[11]. Prasanna G, Guang Y, Ling Z. Primary gastrointenstinal lymphoma. World J Gastroenterol .2011; February 14; 17(6): 697-707.

[12]. Ibrahim.EM, Ezzat AA, Ei-Weshi AN, et al: Primary intestinal diffuse large B-cell non-Hodgkin's lymphoma: clinical features, management, and prognosis of 66 patients. Ann Oncol 2001;12: 53 - 58.

[13]. Radman I, Kovacevic-Metelko J, Aurer I, et al: Surgical resection in the treatment of primary gastrointestinal non-Hodgkin's lymphoma: retrospective study. Croat Med J .2002; 43: 555- 560.

[14]. Samel S, Wagner J, Hofheinz R, et al: Mlignant intestinal non-Hodgkin's lymphoma from the surgical point of view. Onkologie 2002; 25: $268-271$.

[15]. ChulS , Moon J , Pamela K., Lillian M, Fernando C , James D . Primary non-Hodgkin lymphoma of the small bowel. Radiology .1999; 211:183-187. 